\title{
Cluster Calculations of Hyperfine Interactions in Superconducting Copper Compounds
}

\author{
P. F. Meier, T. A. Claxton ${ }^{\text {a }, ~ P . ~ H u ̈ s s e r, ~ S . ~ P l i b e r s ̌ e k, ~ a n d ~ E . ~ P . ~ S t o l l ~}$ \\ Physik-Institut, University of Zürich, Winterthurerstr. 190, CH-8057 Zürich \\ ${ }^{a}$ Department of Chemistry, University of York, York, YO10 5DD, UK \\ Reprint requests to Prof. P. F. M.; E-mail: pfmeier@physik.unizh.ch
}

Z. Naturforsch. 55 a, 247-255 (2000); received August 26, 1999

Presented at the XVth International Symposium on Nuclear Quadrupole Interactions, Leipzig, Germany, July 25 - 30, 1999.

\begin{abstract}
This paper discusses first-principles cluster investigations on the electronic structure of $\mathrm{YBa}_{2} \mathrm{Cu}_{3} \mathrm{O}_{7}$ and $\mathrm{La}_{2} \mathrm{CuO}_{4}$ performed at the University of Zürich. In particular, electric field gradients have been calculated at the copper sites and their dependence on applying uniaxial strain is studied. Magnetic hyperfine interactions have been evaluated from spin-polarized calculations, and the transfer of spin density from neighboring copper ions is explained. An interpretation is given of the nuclear quadrupole resonance spectra in doped $\mathrm{La}_{2} \mathrm{CuO}_{4}$.
\end{abstract}

Key words: Cluster Calculations; Electric Field Gradients; High-Temperature Superconductors; Hyperfine Fields; Nuclear Quadrupole Resonance.

\section{Introduction}

Nuclear quadrupole spectroscopy (NQR) is one of the most productive experimental procedures which non-invasively monitors electron density modifications in high-temperature superconductors with changes in temperature. The method indirectly measures the electric field gradient (EFG) at nuclei with spin $(I>1 / 2)$ and hence sensitively reflects the details of the total electron density distribution in the neighbourhood of these nuclei. Electron density distributions in solids can be calculated using first-principles cluster calculations and an estimate of our confidence in their accuracy can be assessed by calculating the EFG at chosen nuclei and then comparing them with values derived from NQR spectra. Of course once the wave functions have been determined the way is open to the calculation of other physical properties. This should provide a good method of showing which properties are important in deciding superconduction. In this paper we concentrate on two of the simplest materials which exhibit high temperature superconductivity (HTS), $\mathrm{YBa}_{2} \mathrm{Cu}_{3} \mathrm{O}_{7}$ and $\mathrm{La}_{2} \mathrm{CuO}_{4}$. These materials have different structures and composition and should help to elucidate the important features of the electronic structure necessary for superconduction.

The calculated quantities are ground-state properties of the solid and depend sensitively on how charge is distributed within the lattice. Nuclear magnetic resonance (NMR) investigations of the Knight shift and the various nuclear relaxation rate tensors as a function of temperature, doping, and orientation of the applied field with respect to the crystalline axes, provide an additional insight into the static spin density distribution and the low frequency spin dynamics of the electrons in the normal as well as in the superconducting state. Owing to the varied abundances of nuclei with suitable nuclear magnetic and quadrupole moments, most of these quantities can be studied on different sites in the unit cell. This allows one to distinguish static and dynamic features in the $\mathrm{CuO}_{2}$ planes from those in the interlayer region.

Despite the rich information of NQR and NMR results about the nature of the spin fluid and the lowenergy quasiparticles, there exist only few theoretical first-principles approaches which address the determination of electric-field gradients and magnetic hyperfine interactions. Therefore, we have recently car- 
ried out cluster calculations on both the $\mathrm{YBa}_{2} \mathrm{Cu}_{3} \mathrm{O}_{7}$ and the $\mathrm{La}_{2} \mathrm{CuO}_{4}$ system.

The general idea of the cluster approach to electronic structure calculations of properties which depend upon predominantly local electron densities is that the parameters that characterize a small cluster should be transferable to the solid and largely determine its properties. The essential contributions to EFGs and to magnetic hyperfine fields are given by rather localized interactions and therefore it is expected that these local properties can be determined and understood with clusters calculations. Approximations must be made concerning the treatment of the background that is employed to embed the cluster. Using as large a cluster as is possible is of course advantageous. It is necessary, however, that the results obtained should be checked with respect to their dependence on the cluster size.

In this paper, we summarize some of the relevant results and report on new findings about the variation of the EFG at the planar $\mathrm{Cu}$ site in $\mathrm{YBa}_{2} \mathrm{Cu}_{3} \mathrm{O}_{7}$ under application of hydrostatic and uniaxial pressure. Spin-polarized calculations allow us to determine the on-site and transferred hyperfine fields in $\mathrm{La}_{2} \mathrm{CuO}_{4}$ and to compare them with those obtained for the $\mathrm{YBa}_{2} \mathrm{Cu}_{3} \mathrm{O}_{7}$ system. Furthermore, the influence of doping $\mathrm{La}_{2} \mathrm{CuO}_{4}$ has been investigated by replacing a lanthanum atom by a strontium atom as well as by adding an excess oxygen atom.

\section{Cluster Calculations on $\mathrm{YBa}_{2} \mathrm{Cu}_{3} \mathrm{O}_{7}$}

For the $\mathrm{YBa}_{2} \mathrm{Cu}_{3} \mathrm{O}_{7}$ system, EFGs at the various nuclear sites have been obtained by Das and coworkers $[1,2]$ with ab initio cluster calculations using the unrestricted Hartree-Fock (UHF) method, and by Schwarz and coworkers [3, 4] who employed the full-potential linear augmented-planewave (FLAPW) method within the local density approximation (LDA). Results of Hartree-Fock (HF) calculations have also been published by Winter et al. [5]. The EFGs calculated with these three different methods more or less agree and reproduce the experimental data quite satisfactorily apart from one exception, the EFG at the planar $\mathrm{Cu}(2)$ sites.

Using large clusters we recently performed [6] calculations with both the HF and the density functional (DF) method. The largest cluster that we used for the determination of EFGs at the $\mathrm{Cu}(2)$ site is shown in Figure 1. It has point group symmetry $\mathrm{D}_{2 \mathrm{~h}}$ and
Table 1. Theoretical and experimental electric field gradients (in a.u.) and asymmetry parameters at $\mathrm{Cu}(2)$ for $\mathrm{YBa}_{2} \mathrm{Cu}_{3} \mathrm{O}_{7}$.

\begin{tabular}{lcccr}
\hline Method & $V_{a a}$ & $V_{b b}$ & $V_{c c}$ & $\eta$ \\
\hline UHF [2] & & & -2.28 & 0.04 \\
LAPW [3] & 0.31 & 0.27 & -0.58 & 0.07 \\
HF & 1.16 & 0.97 & -2.13 & 0.09 \\
DF-GGA & 0.84 & 0.82 & -1.66 & 0.01 \\
Experiment [13] & $0.643 \pm 0.002$ & $0.628 \pm 0.002$ & $-1.271 \pm 0.002$ & 0.01 \\
\hline
\end{tabular}

consists of 74 atoms: $12 \mathrm{Cu}, 42 \mathrm{O}, 8 \mathrm{Ba}$, and 12 $\mathrm{Y}$. There are 10 planar $\mathrm{Cu}$ and 32 planar $\mathrm{O}$ sites as well as 2 chain $\mathrm{Cu}$ and 10 chain $\mathrm{O}$ sites. The atomic positions were located according to the orthorhombic structure with symmetry $\mathrm{P} 4 / \mathrm{mmm}$ and unit cell parameters $a=3.827, b=3.882$, and $c=11.682 \AA$. In addition, Madelung potentials were included to provide a suitable crystal environment. For the two central $\mathrm{Cu}(2)$ atoms an all-electron basis set originating from Wachters [7] was chosen. For all other $\mathrm{Cu}$ atoms as well as for the $\mathrm{Y}$ and $\mathrm{Ba}$ atoms the pseudopotential of Hay and Wadt [8] with a double zeta basis set was used. The basis for the oxygens was also of double zeta quality [9]. In this way a total number of 556 electrons was accounted for explicitly, whereas pseudopotentials were used for the remaining electrons.

The HF and DF calculations were performed with the Gaussian94 program. The Vosko-Wilk-Nussair functional (VWN) [10] was used as LDA. For the generalized gradient corrections (GGA) the formula proposed by Becke [11] together with the functional of Lee, Yang, and Parr [12] was incorporated (BLYP). All these calculations were non-spin polarized since an appropriate spin-polarized investigation would be extremely complicated. Even in superconducting $\mathrm{YBa}_{2} \mathrm{Cu}_{3} \mathrm{O}_{7}$ antiferromagnetic fluctuations are present. However, they occur very fast on the time scale of NMR measurements and do not influence static properties.

In Table 1 our results for the EFGs of $\mathrm{Cu}(2)$ are summarized and compared with other calculations. For comparison, the experimental values [13, 14] for the quadrupole frequencies were transformed into EFG values assuming a nuclear quadrupole moment $Q\left({ }^{63} \mathrm{Cu}\right)=-0.211 \mathrm{~b}[15]$.

As mentioned previously neither the UHF or FLAPW calculations compare well with experiment and this also applies to our HF calculations. Much better agreement has been obtained with the DF 


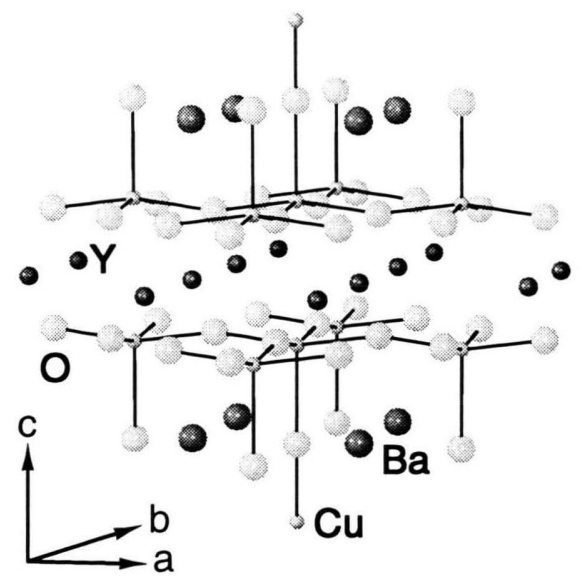

Fig. 1. The $\mathrm{Cu}_{12} \mathrm{O}_{42} \mathrm{Ba}_{8} \mathrm{Y}_{12}$ cluster with $12 \mathrm{Y}$ atoms in the middle plane. $\mathrm{Cu}$ (small) and $\mathrm{O}$ (large) atoms are connected with lines. The remaining dark balls denote the $\mathrm{Ba}$ atoms.

calculations (using the BLYP functional which includes GGA). Note that if we had used the nuclear quadrupole moment of $Q\left({ }^{63} \mathrm{Cu}\right)=-0.18 \mathrm{~b}$, as has been suggested by Stein et al. [16], our DFGGA results would have been even closer to the experimental data. The large differences between the EFG values determined with $\mathrm{HF}$ and DF methods is also observed in cluster calculations on $\mathrm{La}_{2} \mathrm{CuO}_{4}$ (see below).

In the search for an understanding of the pairing mechanism in high $T_{c}$ superconductors, experiments have been carried out on the pressure dependence of various physical quantities. These experiments are very important since they produce controlled variations on the lattice parameters enabling a more detailed study of the specific interactions involved.

The variation of the crystal lattice parameters of $\mathrm{YBa}_{2} \mathrm{Cu}_{3} \mathrm{O}_{7}$ with change of hydrostatic pressure has been measured [17] by neutron diffraction with great accuracy. Our work has now been extended to investigate the influence of pressure on the electronic structure. The calculations are a logical extension of those already published. The positions of the nuclei in the cluster and in the embedding lattice are modified to take account of the lattice parameters at a particular pressure and the EFGs are recalculated. The results obtained with DF and BLYP are displayed in the inset in Fig. 2 and clearly show that the changes calculated correspond closely with those that have been observed experimentally [18]. This is crucial in adding to our confidence in the first-principles calculations since

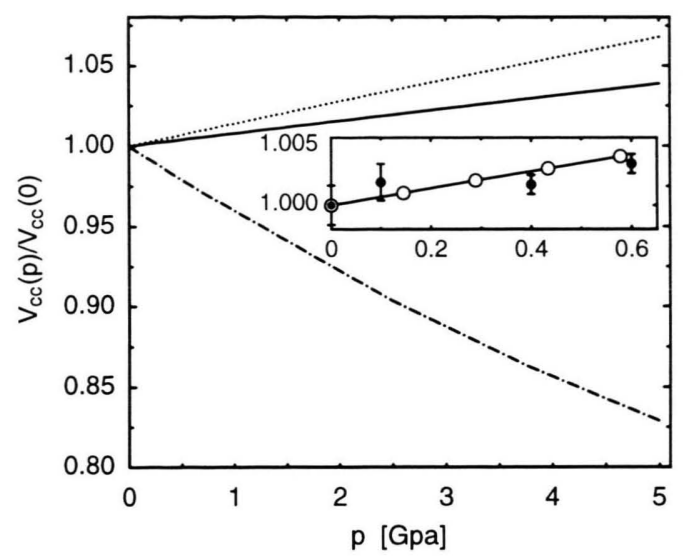

Fig. 2. EFG components $\mathrm{V}_{c c}$ at the $\mathrm{Cu}(2)$ site in the $\mathrm{Cu}_{12} \mathrm{O}_{42} \mathrm{Ba}_{8} \mathrm{Y}_{12}$ cluster versus uniaxial pressure along the axes $a$ (solid), $b$ (dotted), and $c$ (dashed-dotted). The inset shows $V_{c c}$ vs. hydrostatic pressure together with experimental data [18].

trends are perhaps even more important than absolute values.

Intriguing results were obtained from measurements [19] of the change of the superconducting transition temperature $\mathrm{T}_{c}$ upon applying uniaxial pressure which gave $\mathrm{d} T_{c} / \mathrm{d} p_{a}=-2.0 \mathrm{~K} / \mathrm{GPa}$ for strain along the $a$-axis, but $\mathrm{dT}_{c} / \mathrm{dp}_{b}=+2.0 \mathrm{~K} / \mathrm{GPa}$ and $\mathrm{d} T_{c} / \mathrm{d} p_{c}=$ $+0.2 \mathrm{~K} / \mathrm{GPa}$ for the $b$ - and $c$-axis, respectively. This is very surprising since many theoretical models of HTS emphasize interlayer coupling from which it would be expected that strain along the $c$-axis influences $T_{c}$ most strongly. So far there are no experimental results available on the change of lattice parameters upon uniaxial strain. Theoretical information is available from DF calculations that Pickett performed [20] within the FLAPW method. The equilibrium atomic positions were then obtained by minimizing the total energy. It was found that internal strain dominates the changes in the electronic structure. Charge transfer between the chains and the layers was found to be neglible in comparison to the charge rearrangements within the layers themselves which have a strong dipolar character and are directed oppositely for the $a$ - and $b$-axis strain.

We have used these theoretically obtained lattice parameters for uniaxial pressure up to $5 \mathrm{GPa}$ to obtain the wave functions of the occupied cluster orbitals and the EFG in all cases. Unfortunately there is no NQR data available for this system while uniaxial pressure is applied so it is hoped that the results shown in Fig. 2 will stimulate the experimental NQR 


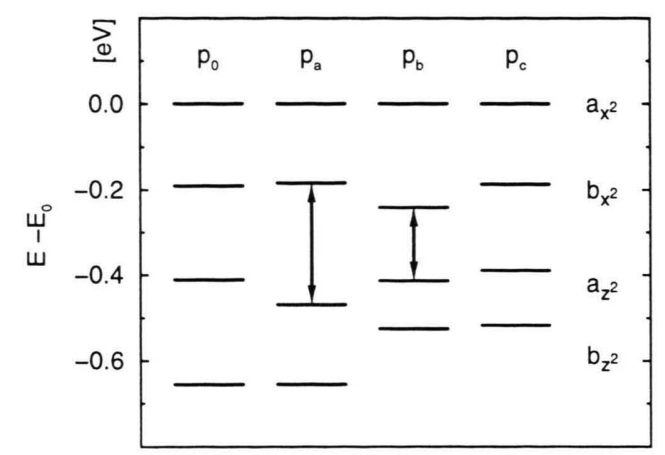

Fig. 3. Relative energies of the four highest occupied MOs as calculated with the DF method for the $\mathrm{Cu}_{12} \mathrm{O}_{42} \mathrm{Ba}_{8} \mathrm{Y}_{12}$ cluster with lattice sites corresponding to zero pressure and uniaxial pressure of $5 \mathrm{GPa}$ along the three axes.

work to be undertaken. However an analysis of the wave function on the copper atoms in the plane has enabled a correlation to be made with the changes in $T_{c}$.

In Fig. 3 we compare the relative energies of the occupied molecular orbitals (MO) as calculated for coordinates corresponding to zero pressure $\left(p_{0}\right)$ and uniaxial pressures of $5 \mathrm{GPa}$ along the three axes $\left(p_{a}\right.$, $p_{b}$, and $p_{c}$ ). In each case, the highest occupied molecular orbital (HOMO) consists of antibonding combinations between the $\mathrm{d}_{x^{2}-y^{2}}$ atomic orbital (AO) of the $\mathrm{Cu}(2)$ atom and the $\mathrm{p}_{x}$ and $\mathrm{p}_{y}$ orbitals of the four neighbouring planar oxygen atoms. The symmetry of this $\mathrm{HOMO}$ is $\mathrm{B}_{1 \mathrm{u}}$ and its energy is called $E_{0}$ and set to zero. The second highest occupied orbital, with energy $-0.17 \mathrm{eV}$ for $p_{0}$, has symmetry $\mathrm{A}_{\mathrm{g}}$. In the former, the orbitals in the two copper planes are antibonding, in the latter, they are bonding. We denote them $\mathrm{a}_{x^{2}}$ and $\mathrm{b}_{x^{2}}$. The third and fourth MO are antibonding combinations between the $3 \mathrm{~d}_{z^{2}}$ of $\mathrm{Cu}$ and the $2 \mathrm{p}_{z}$ of the apex oxygens and antibonding $\left(\mathrm{a}_{z^{2}}\right)$ and bonding $\left(b_{z^{2}}\right)$ between the planes. From an inspection of Fig. 3 it is seen that uniaxial strain along different axes has a drastic effect on the energy differences between these four MO. The separation between $\mathrm{b}_{x^{2}}$ and $\mathrm{a}_{z^{2}}$, in particular, which is $220 \mathrm{meV}$ for zero pressure, is strongly increased to $284 \mathrm{meV}$ for $p_{a}$, slightly reduced to $203 \mathrm{meV}$ for $p_{c}$, but markedly reduced to $172 \mathrm{meV}$ for $p_{b}$. This correlates with an increased (decreased) hole character of the $\mathrm{Cu} 3 \mathrm{~d}_{z^{2}}$ AOs for pressure along the $b(a)$ axis. These changes in the calculated electronic structure are under further study. Similar effects have been observed by investi-

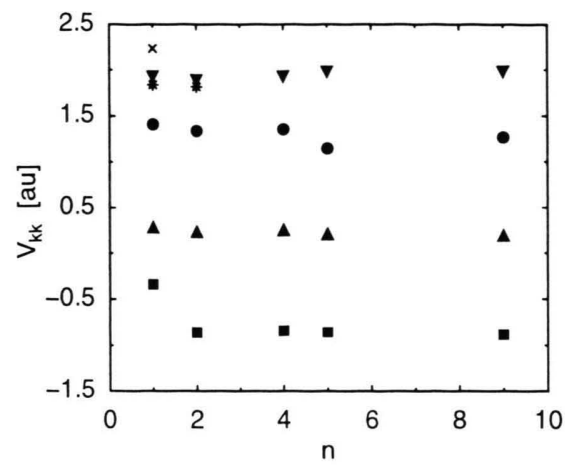

Fig. 4. $V_{k k}$ at central copper sites plotted against $n$, the number of copper atoms in the clusters $\mathrm{Cu}_{n} \mathrm{O}_{m}$ modelling the $\mathrm{La}_{2} \mathrm{CuO}_{4}$ system. HF results: $\boldsymbol{\nabla}=V_{c c}(\mathrm{Cu}), \times:$ : 21], *: [23]. DF results: $\bullet=V_{c c}(\mathrm{Cu}), \boldsymbol{\Delta}=V_{c c}(\mathrm{O}(\mathrm{a})), \mathbf{\square}=V_{a a}(\mathrm{O}(\mathrm{p}))$.

gating the effects of doping in the La compound and will be discussed below.

\section{Cluster Calculations on $\mathrm{La}_{2} \mathrm{CuO}_{4}$}

For the $\mathrm{La}_{2} \mathrm{CuO}_{4}$ system, UHF cluster calculations have been reported by Sulaiman et al. [21] who obtained EFG values at various nuclei as well as the contact and dipolar hyperfine fields at $\mathrm{Cu}$. Martin and Hay [22] presented HF results of the electronic structure of $\mathrm{CuO}_{6}$ clusters in neutral, electron- and holedoped states and studied the influence of correlation effects using the technique of configuration interactions. Martin [23] calculated HF values for EFGs in $\mathrm{CuO}_{6}$ and $\mathrm{Cu}_{2} \mathrm{O}_{11}$ clusters and investigated the change in the NQR spectra upon doping. A comparison [24] of results obtained with HF and LDA calculations for a $\mathrm{CuO}_{6}$ cluster again showed large differences in the EFG values and the hyperfine coupling parameters.

Recently, we have performed [25, 26] extensive cluster studies for the $\mathrm{La}_{2} \mathrm{CuO}_{4}$ system. Spinpolarized calculations at the $\mathrm{HF}$ level and with the DF method with gradient corrections to the correlation functionals have been carried out for clusters $\mathrm{Cu}_{n} \mathrm{O}_{m}$ comprising $n=1,2,4,5$, and 9 copper atoms in a plane. As in our investigations on $\mathrm{YBa}_{2} \mathrm{Cu}_{3} \mathrm{O}_{7}$, we used the BLYP functionals to account for exchange and correlation effects. The atomic positions were located according to the tetragonal phase. The clusters were embedded in a lattice of more than 2000 point charges. Some positions of them were adjusted to get the correct Madelung potential in the central region of the cluster. Bare pseudopotentials instead of point 


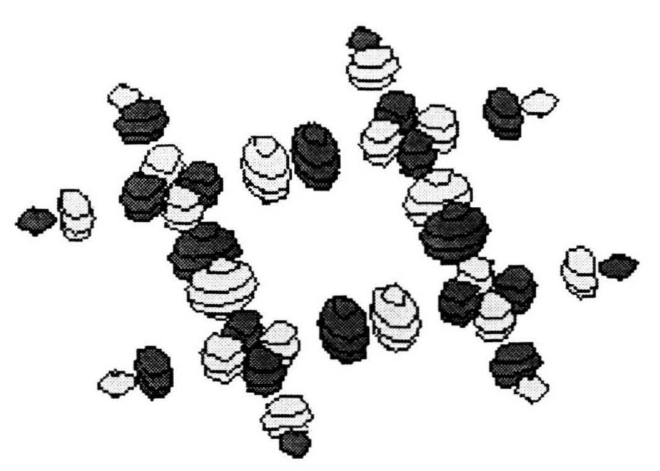

Fig. 5. Highest occupied molecular orbital for the $\mathrm{Cu}_{4} \mathrm{O}_{20}$ cluster modelling the $\mathrm{La}_{2} \mathrm{CuO}_{4}$ system.

charges were used for the positive $\mathrm{Cu}$ - and $\mathrm{La}$ - ions closest to the cluster border. For the cluster atoms, a 6-311G basis set was used. The resulting electronic structures, the charge and spin distributions, EFGs and magnetic hyperfine interactions have been analyzed.

In Fig. 4 we display the results for the EFG components $V_{k k}$ at various nuclear sites as a function of the cluster size. Consider first the upper two sets of data that refer to the HF (triangles-down) and DF (circles) results for $V_{c c}$ at the copper. While the convergences with increasing number of cluster copper atoms are reasonably well, large differences are to be noted between the values obtained with HF and DF calculations. Our HF results for small clusters ( $n=1$ and 2 ) are in broad agreement with those obtained by Sulaiman et al. [21] (cross) and Martin [23] (stars). The experimental value [28] for the quadrupole frequency is ${ }^{63} \nu_{\mathrm{Q}}=33.0 \mathrm{MHz}$ as measured at a temperature of $600 \mathrm{~K}$ in the high temperature tetragonal phase. This corresponds to an EFG component $V_{c c}=1.33 \mathrm{a}$. u. assuming a quadrupole moment $Q\left({ }^{63} \mathrm{Cu}\right)=-0.211 \mathrm{~b}$ as proposed in [15] but $V_{c c}=1.56$ a. u. for $Q\left({ }^{63} \mathrm{Cu}\right)=$ $-0.18 \mathrm{~b}$ as calculated in [16]. Next we discuss the lower two sets of data that refer to the DF results for $V_{c c}$ at the apical oxygen, $\mathrm{O}$ (a) (triangles-up), and for $V_{a a}$ at the planar oxygen site $\mathrm{O}(\mathrm{p})$ (squares). At the ${ }^{17} \mathrm{O}(\mathrm{p})$, the measured [27] quadrupole frequency is $0.69 \mathrm{MHz}$ with an asymmetry parameter of $\eta=0.36$. Our results for the largest cluster which contains nine copper atoms render ${ }^{17} \nu_{\mathrm{Q}}=0.46 \mathrm{MHz}$ and $\eta=0.25$. The experimental value for ${ }^{17} \mathrm{O}$ (a) corresponds to $V_{c c}$ $=0.22 \mathrm{a}$. u.. The agreement between these experimental values and our DF results is reasonable considering that our clusters were constructed to mainly investi-

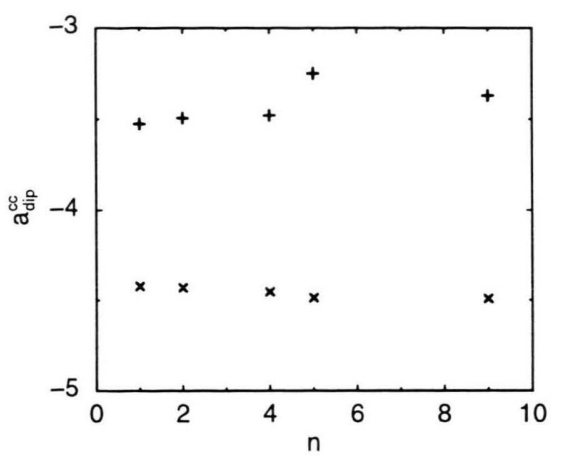

Fig. 6. $a_{\text {dip }}^{c c}(\mathrm{Cu})$ in atomic units plotted against $n$, the number of copper atoms in clusters $\mathrm{Cu}_{n} \mathrm{O}_{m}$ modelling the $\mathrm{La}_{2} \mathrm{CuO}_{4}$ system. Symbols $+(\times)$ refer to $\mathrm{DF}(\mathrm{HF})$ results.

gate the copper EFG.

All our results were obtained for the spin states with highest multiplicity. In Fig. 5 we show the highest occupied molecular orbital for the cluster $\mathrm{Cu}_{4} \mathrm{O}_{20}$.

As concerns the magnetic hyperfine interactions, the analysis of many NQR and NMR experiments that were carried out immediately after the discovery of HTS was puzzling. Mila and Rice [29] proposed then that besides the usual on-site hyperfine interactions at a $\mathrm{Cu}$ nucleus an additional hyperfine field should be considered which is transferred from neighboring $\mathrm{Cu}$ ions. They gave a consistent explanation of the unusual combination of anisotropies of the $\mathrm{Cu}$ Knight shifts and relaxation rates in $\mathrm{YBa}_{2} \mathrm{Cu}_{3} \mathrm{O}_{7}$ within a one-component theory. An estimation of the strength of the various hyperfine parameters with the use of a quantum chemical model led to good agreement with the values deduced from experiment.

Using the data from our series of clusters with increasing number of copper atoms, we have investigated the magnetic hyperfine interactions in $\mathrm{La}_{2} \mathrm{CuO}_{4}$ in depth.

The total magnetic hyperfine interaction, $A(\mathrm{X})$, at a nucleus $\mathrm{X}$, arises from the interaction of the nuclear spin of $\mathrm{X}$ with electron spins dispersed according to the spin density distribution. It consists of two components, a scalar contact term, $D(\mathrm{X})$, and a tensor dipolar term, $a_{\mathrm{dip}}^{k l}(\mathrm{X})$, where $k$ and $l$ refer to crystal axes $a, b$ or $c$, that is,

$$
A^{k l}(\mathrm{X})=D(\mathrm{X})+a_{\mathrm{dip}}^{k l}(\mathrm{X}) .
$$

$D(\mathrm{X})$ is proportional to the spin density at the nucleus $\mathrm{X}$ and $a_{\mathrm{dip}}^{k l}(\mathrm{X})$ is the classical dipole-dipole interaction of nuclear spin $\mathrm{X}$ with the electron spin distribution. 


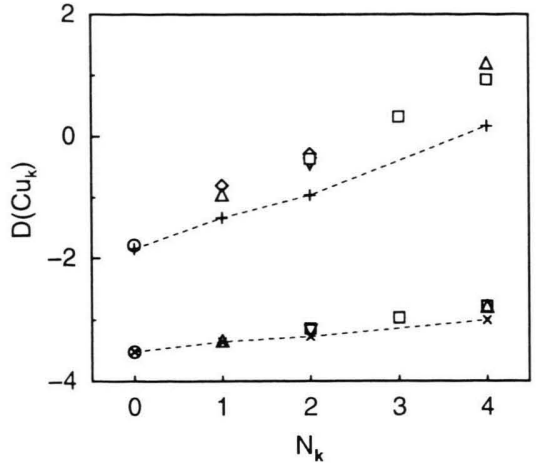

Fig. 7. Difference between spin-up and spin-down densities, $D\left(\mathrm{Cu}_{k}\right) v s$. $N_{k}$, the number of copper atoms that are nearest neighbors to $\mathrm{Cu}_{k}$, as calculated for various clusters containing $n \mathrm{Cu}$ atoms. For clusters representing $\mathrm{La}_{2} \mathrm{CuO}_{4}$ the values of $n$ are $\diamond=1, \diamond=3, \nabla=4, \Delta=5, \square=9$. DF (HF) values correspond to the upper (lower) data set. For clusters representing $\mathrm{YBa}_{2} \mathrm{Cu}_{3} \mathrm{O}_{7}$ the values are represented by + for DF and $\times$ for HF calculations. To aid visuality, the + and $x$ points are connected by lines.

In Fig. 6 the results for the dipolar hyperfine interaction parameter $a_{\text {dip }}^{c c}(\mathrm{Cu})$ are shown as a function of the cluster size. In terms of the model for a single hole on the $\mathrm{Cu}^{2+}$ ion, developed by Bleaney et al. [30], this coupling is given by $-4 / 7\left\langle r^{-3}\right\rangle$. Our DF (HF) values then would correspond to an average $\left\langle r^{-3}\right\rangle \approx$ 6.0 (7.8) a. u..

As concerns the core-polarization term $D(\mathrm{Cu})$, it turned out that the values strongly depend on the position of a particular copper atom in larger clusters. This is illustrated in Fig. 7 where $D\left(\mathrm{Cu}_{k}\right)$ is plotted versus $N_{k}$, the number of copper atoms that are nearest neighbors of $\mathrm{Cu}_{k}$. It is seen that $D\left(\mathrm{Cu}_{k}\right)$ increases linearly with $N_{k}$ for both DF and HF methods and can thus be separated into

$$
D\left(\mathrm{Cu}_{k}\right)=a+b N_{k},
$$

i.e., into an on-site core-polarization term $a$ and a transferred hyperfine field $b$. From a detailed analysis of all calculations (some of them also for lower spinmultiplicities) we obtained the DF values $a=-1.78$ and $b=0.71$. There is also a small transferred dipolar hyperfine field, $b_{\text {dip }}^{c c}=0.07$, which explains the slight increase of the total $a_{\text {dip }}^{c c}(\mathrm{Cu})$ (see Fig. 6) when $N_{k}$ becomes larger.

Recently we were also in a position to carry out [31] spin-polarized calculations for the $\mathrm{YBa}_{2} \mathrm{Cu}_{3} \mathrm{O}_{7}$ system. To this end, a cluster was chosen by dropping the

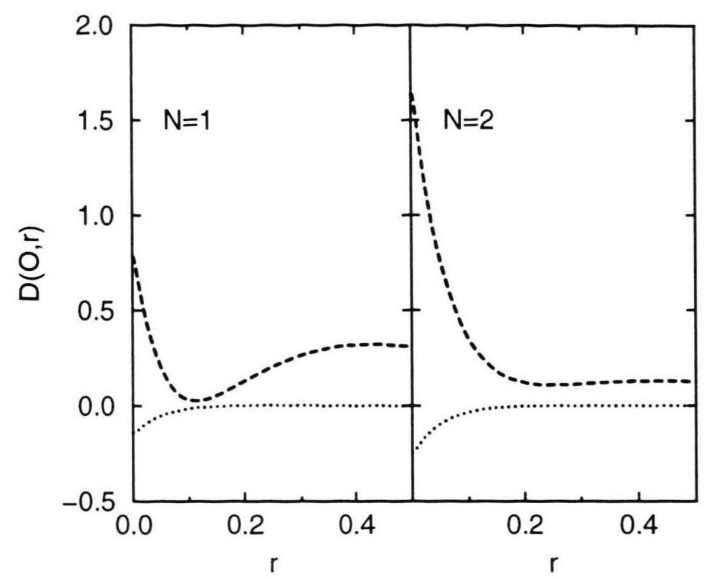

Fig. 8. Radial dependence of the difference between spin-up and -down densities for a planar oxygen with one $(N=1)$ nearest copper atom $\left(\mathrm{CuO}_{6}\right)$ and for an oxygen bridging two $(N=2)$ copper atoms $\left(\mathrm{Cu}_{2} \mathrm{O}_{11}\right)$ in the $2 \mathrm{~s}$ (dashed) and 1s (dotted) atomic orbital.

lower half of the cluster shown in Figure 1. To be precise, it contained five $\mathrm{Cu}$ atoms in the $\mathrm{CuO}_{2}$ plane and $21 \mathrm{O}$ atoms treated with an all-electron $6-311 \mathrm{G}$ basis set. In addition, some positive point charges close to the cluster boundary $(11 \mathrm{Cu}, 12 \mathrm{Y}, 4 \mathrm{Ba})$ were represented by bare pseudopotentials. The resulting dependencies of the core-polarization term $D\left(\mathrm{Cu}_{k}\right)$ on $N_{k}$ are also shown in Figure 7. The linear increase of $D\left(\mathrm{Cu}_{k}\right)$ with $N_{k}$ is again observed with an on-site and transferred hyperfine parameter of $a=-1.7$ and $b=0.5$, respectively.

In retrospective, some of the early problems in analyzing NQR and NMR data on cuprate superconductors can be traced back to the fact that the single-hole model developed in [30] for an isolated $\mathrm{Cu}^{2+}$ ion in a crystal field, cannot be applied to correlated $\mathrm{Cu}$ ions in a $\mathrm{CuO}_{2}$ plane. It works certainly for local quantities like the dipolar coupling tensor which are mainly determined by the HOMO and also do not depend too sensitively on the method of treating the manyelectron system. Even HF calculations give results of the correct order of magnitude. The core-polarization, however, is not confined to the atom in question. As Fig. 7 demonstrates, hyperfine fields transferred from the neighboring copper ions have a drastic effect on $D(\mathrm{Cu})$. Its value is negative for an isolated copper ion but is increased by contributions from neighboring ions. At the HF level, there is a small hybridization and the transfer is small. DF calculations, however, render a stronger covalency and therefore the spin 


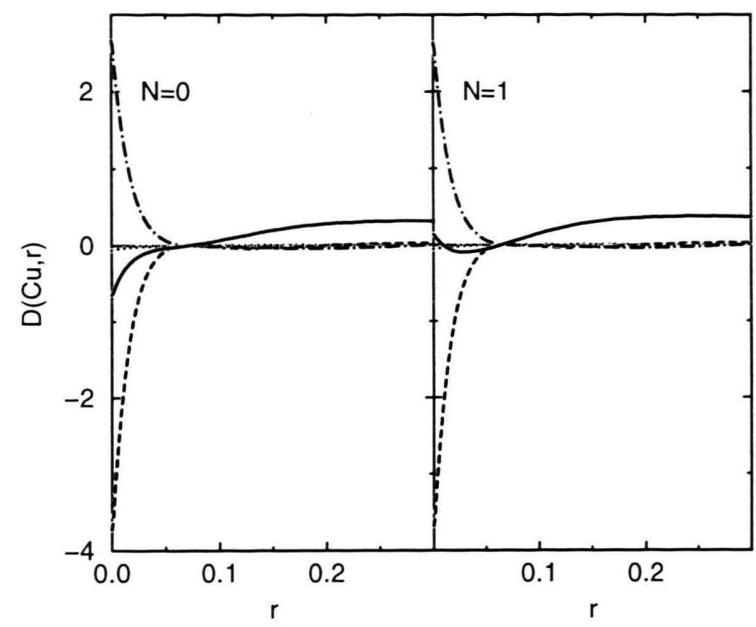

Fig. 9. Radial dependence of the difference between spinup and -down densities at a copper atom with none $(N=0)$ nearest copper atom and for one $(N=1)$ nearest neighbor copper in the $4 \mathrm{~s}$ (solid), 3s (dashed-dotted), $2 \mathrm{~s}$ (dashed) and $1 \mathrm{~s}$ (dotted) atomic orbital.

density is more delocalized which implies a larger transfer. With contributions from four nearest neighbor $\mathrm{Cu}$ ions, $D(\mathrm{Cu})$ becomes positive as was first recognized by Mila and Rice [29].

To investigate the mechanisms of spin transfer, we will analyze in detail the DF results from the two clusters containing one and two copper atoms and point out the differences. Let us first consider the $\left(\mathrm{CuO}_{6}\right)^{10-}$ ion embedded in the appropriate lattice background. Due to overlap and covalent effects the $\mathrm{Cu}^{2+}$ ion shares its spin density with the ligand planar oxygens whose spin direction is parallel to that of the local $\mathrm{Cu}$ moment. The atomic spin density $\rho(\mathrm{Cu})$ is reduced from 1 to 0.667 in favor of a spin density transfer to the four planar oxygens $(4 \times \rho(\mathrm{O}(\mathrm{p}))$ $=0.328)$. The transferred spin density is mainly on the $\mathrm{O} 2 \mathrm{p}_{\sigma}$ orbital, but a small amount also goes to the $\mathrm{O} 2 \mathrm{~s}$ which is thus expected to be polarized parallel to the spin density on the $\mathrm{Cu}$. Indeed, we get $D_{2 s}(\mathrm{O})=0.772$. The $2 \mathrm{~s}$ orbital in turn polarizes the $1 \mathrm{~s}$ but with opposite sign $\left(D_{1 \mathrm{~s}}(\mathrm{O})=-.141\right)$. This is shown in the left panel of Figure 8.

On the $\mathrm{Cu}$, the difference of spin densities of the $4 \mathrm{~s}$ orbitals is also positive. Close to the nucleus, however, it becomes negative with $D_{4 \mathrm{~s}}(\mathrm{Cu})=-.650$ (see Figure 9). The $4 \mathrm{~s} \mathrm{AO}$ in turn polarizes the inner $\mathrm{s}$ orbitals. The alternating signs of the $4 \mathrm{~s}, 3 \mathrm{~s}$, and $2 \mathrm{~s}$ contributions can qualitatively be explained as a direct consequence of Hund's rule.

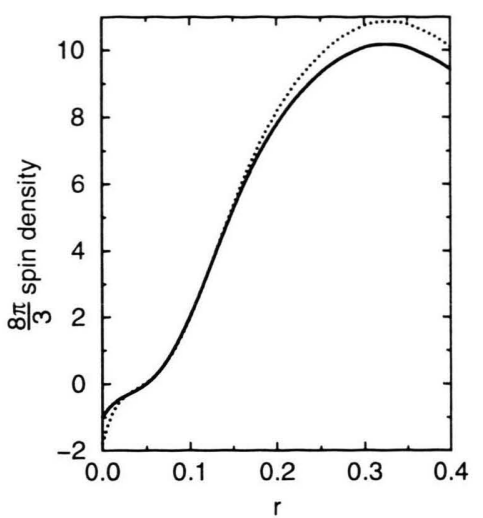

Fig. 10. Radial dependence of the total spin density difference at a copper atom without other $\mathrm{Cu}$ (dotted) and with one nearest neighbour $\mathrm{Cu}$ (solid).

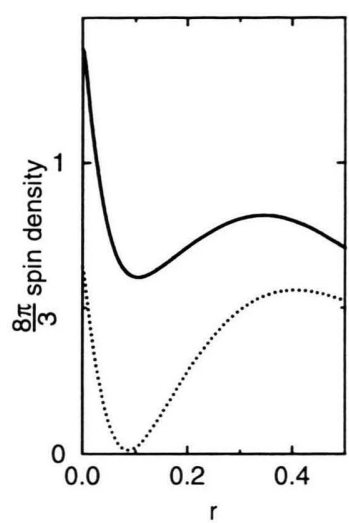

Fig. 11. Radial dependence of the total spin density difference at an oxygen with one neighboring $\mathrm{Cu}$ (dotted) and with two nearest neighbour $\mathrm{Cu}$ (solid).

We next consider the cluster $\mathrm{Cu}_{2} \mathrm{O}_{11}$ consisting of $2 \mathrm{Cu}$ atoms with parallel moments and with 1 bridging $\mathrm{O}\left(\mathrm{O}_{\mathrm{br}}\right)$ and 6 planar $\mathrm{O}\left(\mathrm{O}_{1}\right)$ bonded to one of the $\mathrm{Cu}$ only. The DF calculations yield the atomic spin densities $\rho(\mathrm{Cu})=0.670, \rho\left(\mathrm{O}_{\mathrm{br}}\right)=0.139$, and $\rho\left(\mathrm{O}_{1}\right)=0.092$. The changes in the s-like AOs can be seen by comparing the right and left panels in Figs. 8 and 9.

The radial dependences of the difference of the total spin densities (which for convenience have been multiplied by $8 \pi / 3$ ) are shown in Figs. 10 and 11 .

\section{Hole Doped $\mathrm{La}_{2} \mathrm{CuO}_{4}$}

$\mathrm{La}_{2} \mathrm{CuO}_{4}$ becomes superconducting if a sufficient number of holes is introduced into the $\mathrm{CuO}_{2}$ planes 
either by alloying on the La site or by changing the oxygen content. Understanding the changes in the local electronic structure, which are produced by these impurities, is therefore very desirable. As mentioned above, the NQR spectra [28] for stoichiometric $\mathrm{La}_{2} \mathrm{CuO}_{4}$ exhibit one narrow line (linewidth $55 \mathrm{kHz}$ ) at about $33 \mathrm{MHz}$ assigned to ${ }^{63} \mathrm{Cu}$. In $\mathrm{La}_{2-x} \mathrm{Sr}_{x} \mathrm{CuO}_{4}$, an additional peak which was called $\mathrm{B}$-line is observed at a somewhat higher frequency [32] whereas the main resonance line (A) turns out to be much broader $(\approx 1 \mathrm{MHz})$ than in the undoped case. With increasing dopand concentration $x$ the frequencies of both peaks increase and the intensity of the B-line grows. Also when excess oxygen is added, the NQR spectra [33] show a similar behavior to those in Sr-doped materials. The frequency of the satellite peak in $\mathrm{La}_{2} \mathrm{CuO}_{4+\delta}$ is, for comparable values of $x$ and $\delta$, so close to that of the B-line in Sr-doped samples that both lines were thought to have identical physical origins, namely localized holes. These would then give rise to practically identical frequencies for the B-lines in Sr- and O- doped $\mathrm{La}_{2} \mathrm{CuO}_{4}$. To be responsable for the NQR spectra, these holes would be required, however, to be localized for time scales of microseconds. Additional support for this interpretation came from $a b$ initio cluster calculations of the EFG by Martin [23]. In his HF approach the EFG at the copper site changes too little $(1.5 \%)$ when a $\mathrm{Sr}$ atom replaced the La but too much (14\%) for excess $\mathrm{O}$ to explain the NQR data. The calculated change in the EFG at a $\mathrm{Cu}$ site next to a localized hole, however, was in good agreement with the experimentally observed frequency difference $(\approx 10 \%)$ between the $\mathrm{A}-$ and B-lines.

Encouraged by our results on the dependence of the EFG on the cluster size discussed in the previous section, we have investigated [34] the doping dependence with our $\mathrm{CuO}_{6}$ cluster surrounded with $4 \mathrm{Cu}$ and $10 \mathrm{La}$ atoms. Exchanging the pseudopotential for the $\mathrm{La}$ atom on top of the $\mathrm{Cu}$ by one for $\mathrm{Sr}$, a situation is modelled where no additional holes are present in the cluster region. The DF results for the copper EFG turned out to be $10 \%$ higher than in the undoped cluster in close agreement with the experimental results for the quadrupole frequencies.

Next we extended the $\mathrm{CuO}_{6}$ cluster by adding an interstitial oxygen $\mathrm{O}$ (i) at the site determined by neutron diffraction. The relaxation of the four oxygen atoms that are nearest neighbours to $\mathrm{O}$ (i) was accounted for by minimizing the total energy. With these atomic

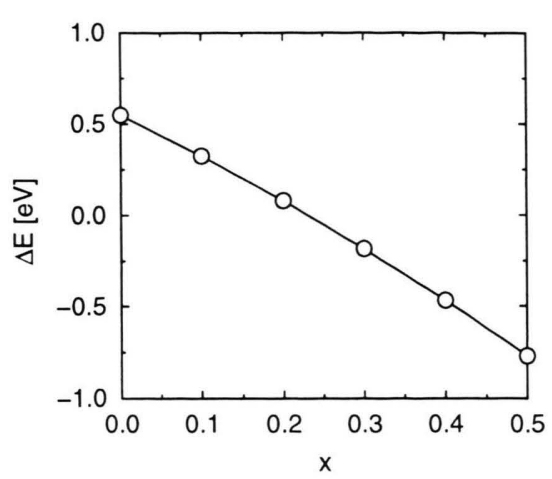

Fig. 12. Difference in energies between the highest occupied MOs with predominantly $3 \mathrm{~d}_{x^{2}-y^{2}}$ and $3 \mathrm{~d}_{z^{2}}$ character versus the doping concentration $x$ calculated with the $\mathrm{CuO}_{6}$ cluster.

positions, the $\mathrm{Cu}$ EFG in the cluster $\mathrm{CuO}_{7}$ was determined to be $11 \%$ larger than in the undoped system. Thus, according to our DF calculations, the similarity between the NQR spectra of $\mathrm{La}_{2-x} \mathrm{Sr}_{x} \mathrm{CuO}_{4}$ and $\mathrm{La}_{2} \mathrm{CuO}_{4+\delta}$ is accidental, and there is no need to advocate localized holes for the interpretation.

We also accounted [26, 34] for holes moving rapidly across the plane by a simple model where positive point charges with values $x / 2$ were put close to the four planar oxygen sites. The EFG at the $\mathrm{Cu}$ then linearly increased with $x$ with a slope approximately corresponding to the observed shift of the A-line. Interestingly, along with this change of the EFG upon increasing $x$, the difference in energy levels between the highest occupied orbitals shrinks in accordance with the results discussed above (Fig. 3) for the dependence of the EFG in $\mathrm{YBa}_{2} \mathrm{Cu}_{3} \mathrm{O}_{7}$ for uniaxial pressure along the $b$-axis. This is shown in Fig. 12 where the difference in energies $\Delta E$ between the two highest occupied MOs with spin up (which have predominantly $3 \mathrm{~d}_{x^{2}-y^{2}}$ and $3 \mathrm{~d}_{z^{2}}$ character) is plotted as a function of the point charge strength $x$ that simulates the effect of doping. It is seen that a gradual change in $\Delta E$ takes place. Beyond $x \approx 0.2$ the energy of the $3 d_{z^{2}}$ is higher than that of the $3 d_{x^{2}-y^{2}}$. This is a quantitative confirmation of the estimates in [35].

In addition, the structure of the highest occupied MOs is also changing when comparing the results between the undoped and the $\mathrm{Sr}$ - doped $\mathrm{CuO}_{6}$ cluster. Again, the $\mathrm{MO}$ which predominantly consists of $\mathrm{Cu}$ $3 \mathrm{~d}_{z^{2}}$ and $\mathrm{O}$ (a) $2 \mathrm{p}_{z}$ AOs rises in energy when compared to the $\mathrm{MO}$ with $\mathrm{Cu} 3 \mathrm{~d}_{x^{2}-y^{2}}$ and $\mathrm{O}(\mathrm{p}) 2 \mathrm{p}_{\sigma}$ character.

This change of the ordering of the molecular orbitals upon doping is an important observation which 
deserves further study. It involves a conversion from a strictly planar towards a more three-dimensional behaviour that occurs upon doping. A detailed study of these effects is in progress.

The importance of the apex oxygen is also seen in some experiments. Thus in recent polarizationdependent near-edge $\mathrm{x}$-ray absorption spectroscopic measurements on $\mathrm{Y}_{1-x} \mathrm{Ca}_{x} \mathrm{Ba}_{2} \mathrm{Cu}_{3} \mathrm{O}_{7-y}$ [36], the unoccupied electronic structure of the $\mathrm{CuO}_{2}$ planes has

[1] T. P. Das in "Electronic Properties of Solids Using Cluster Methods", ed. by T. A. Kaplan and S. D. Mahanti, Plenum Publishing Corporation, New York 1995.

[2] N. Sahoo, S. Markert, T. P. Das, and K. Nagamine, Phys. Rev. B 41, 220 (1990).

[3] K. Schwarz, C. Ambrosch-Draxl, and P. Blaha, Phys. Rev. B 42, 2051 (1990).

[4] C. Ambrosch-Draxl, P. Blaha, and K. Schwarz, Phys. Rev. B 44, 5141 (1991).

[5] N. W. Winter, C. I. Merzbacher, and C. E. Violet, Appl. Spec. Rev. 28, 123 (1993).

[6] P. Hüsser, E. P. Stoll, H. U. Suter, and P. F. Meier, Physica C 294, 217 (1998).

[7] A. J. H. Wachters, J. Chem. Phys. 52, 1033 (1970).

[8] P. J. Hay and W. R. Wadt, J. Chem. Phys. 82, 270 (1985).

[9] T. H. Dunning and P. J. Hay, Methods of Electronic Structure Theory, ed. H.F. Schäfer, Plenum Press, New York 1977.

[10] S. H. Vosko, L. Wilk, and M. Nussair, Can. J. Phys. 58, 1200 (1980).

[11] A. D. Becke, Phys. Rev. A 38, 3098 (1988).

[12] C. Lee, W. Yang, and R. G. Parr, Phys. Rev. B 37, 785 (1988).

[13] C. H. Pennington, D. J. Durand, C. P. Slichter, J. P. Rice, E. D. Bukowski, and D. M. Ginsberg, Phys. Rev. B 39, 2902 (1989).

[14] For the comparison, a definite sign for the experimental values was chosen.

[15] R. M. Sternheimer, Z. Naturforsch. 41a, 35 (1985).

[16] J. Stein, S. B. Sulaiman, N. Sahoo, and T. P. Das, Hyperfine Interactions 60, 849 (1990).

[17] J. D. Jorgensen et al., Physica C 171, 93 (1990).

[18] K. Müller, M. Mali, J. Roos, and D. Brinkmann, Physica C 162-164, 173 (1989).

[19] C. Meingast et al., Phys. Rev. Lett. 67, 1634 (1991); U. Welp et al., Phys. Rev. Lett. 69, 2130 (1992). been studied. Unlike the conventional picture where superconductivity is related predominantely to the number of planar holes, clear evidence for a distinguished role of the apical sites is found.

We gratefully acknowledge the contributions of O. Dijkstra, H. U. Suter, and S. Schafroth. We would like to thank D. Brinkmann, M. Mali, and J. Roos for enlightening discussions. This work was partially supported by the Swiss National Science Foundation.

[20] W. E. Pickett, Phys. Rev. Lett. 78, 1960 (1997).

[21] S. B. Sulaiman, N. Sahoo, T. P. Das, and O. Donzelli, Phys. Rev. B 45, 7383 (1992).

[22] R. L. Martin and P. J. Hay, J. Chem. Phys. 98, 8680 (1993).

[23] R. L. Martin, Phys. Rev. Lett. 75, 744 (1995).

[24] H. U. Suter, P. Hüsser, E. P. Stoll, S. Schafroth, and P. F. Meier, Hyperfine Interactions, in press.

[25] P. Hüsser, H. U. Suter, E. P. Stoll, and P. F. Meier, Phys. Rev. B, submitted.

[26] P. Hüsser, Dissertation, Universität Zürich (1999).

[27] K. Ishida et al., J. Phys. Soc. Japan 60, 3516 (1991).

[28] T. Imai, C. P. Slichter, K. Yoshimura, and K. Kosage, Phys. Rev. Lett. 70, 1002 (1993).

[29] F. Mila and T. M. Rice, Physica C 157, 561 (1989).

[30] B. Bleaney, K. D. Bowers, and M. H. L. Pryce, Proc. Roy. Soc. London, Ser. A 228, 166 (1955).

[31] O. Dijkstra et al., to be published

[32] K. Yoshimura, T. Imai, T. Shimuzu, Y. Ueda, K. Kosuge, and H. Yosuoka, J. Phys. Soc. Japan 58, 3057 (1989); Y. Nakamura and K. Kumagai, Physica C 165/166, 1303 (1990); K. Yoshimura, T. Uemura, M. Kato, K. Kosuge, T. Imai, and H. Yasuoka, Hyperfine Interactions 79, 867 (1993); Y.-Q. Song, M. A. Kennard, M. Lee, K. R. Poeppelmeier, and W. P. Halperin, Phys. Rev. B 57, 712 (1998).

[33] P. C. Hammel, A. P. Reyes, S.-W. Cheong, Z. Fisk, and J. E. Schirber, Phys. Rev. Lett. 71, 440 (1993); B. W. Statt, P. C. Hammel, Z. Fisk, S.-W. Cheong, F. C. Chou, D. C. Johnston, and J. E. Schirber, Phys. Rev. B 52, 15575 (1995).

[34] S. Pliberšek and P. F. Meier, submitted.

[35] D. I. Khomskii and E. I. Neimark, Physica C 173, 342 (1991).

[36] M. Merz, N. Nücker, P. Schweiss, S. Schuppler, C. T. Chen, V. Chakarian, J. Freeland, Y. U. Idzerda, M. Kläser, G. Müller-Vogt, and Th. Wolf, Phys. Rev. Lett. 80, 5192 (1998). 